\title{
Influência do estado nutricional no risco para transtornos alimentares em estudantes de nutrição
}

\author{
Influence of the nutritional status in the risk of eating disorders \\ among female university students of nutrition: eating patterns \\ and nutritional status
}

Janiara David Silva ${ }^{1}$

Amanda Bertolini de Jesus Silva ${ }^{2}$

Aihancreson Vaz Kirchoff de Oliveira ${ }^{3}$

Aline Silva de Aguiar Nemer ${ }^{1}$

${ }^{1}$ Departamento de Nutrição, Instituto de Ciências Biológicas, Universidade Federal de Juiz de Fora. Cidade Universitária. 36036-900 Juiz de Fora MG. aline.nemer@uff.edu.br ${ }^{2}$ Escola de Nutrição da Universidade Federal de Ouro Preto.

${ }^{3}$ Faculdade de Medicina, Universidade Federal de Ouro Preto.

\begin{abstract}
The scope of this paper was to evaluate the relationship between changes in eating behavior associated with dissatisfaction with body image, and the nutritional status of female university students of nutrition. A cross-sectional study was conducted among 175 female students of nutrition (ENUT/UFOP). The Eating Attitudes Test26 (EAT-26) and Body Shape Questionnaire (BSQ) were applied and anthropometric measurements were taken. $21.7 \%$ of the students were found to be high risk in terms of eating disorders, and $13.7 \%$ declared dissatisfaction with their body image. The majority of students with positive results in the BSQ and EAT-26 tests were eutrophic. The students who were overweight, with elevated body fat percentage (\% BF) and waist circumference (WC) had a 5-9 times greater risk of change in eating habits. There was a positive association between the anthropometric parameters with high scores in the EAT-26 and BSQ questionnaires. The future dietitians who are overweight, with increased body fat and waist circumference were more likely to be dissatisfied with their body image and develop eating disorders. The use of other anthropometric parameters, in addition to BMI, may prove useful in screening individuals susceptible to the emergence of excessive concerns with weight and diet.
\end{abstract}

Key words Body image, Eating disorders, Nutritional assessment
Resumo Avaliar a relação entre a alteração do comportamento alimentar, associado à insatisfação com a imagem corporal, e o estado nutricional de universitárias de Nutrição. Realizou-se um estudo transversal com 175 universitárias do Curso de Nutrição (ENUT/UFOP). Foram aplicados os questionários Eating Attitudes Test-26 (EAT-26) e o Body Shape Questionare (BSQ) e realizadas medidas antropométricas. $21,7 \%$ das estudantes apresentaram alto risco para transtornos alimentares e 13,7\% apresentaram insatisfação com a imagem corporal. A maioria das estudantes com BSQ e EAT-26 positivos estava eutrófica. As estudantes com excesso de peso, aumento do percentual de gordura corporal (\% GC) e do perímetro da cintura (PC) apresentaram risco 5 a 9 vezes maiores de alteração do comportamento alimentar. Houve associação positiva entre os parâmetros antropométricos com a pontuação dos questionários EAT-26 e BSQ. Futuras nutricionistas com excesso de peso apresentaram maior risco de estarem insatisfeitas com a sua imagem corporal e de desenvolverem transtornos alimentares. A utilização de outros parâmetros antropométricos, além do IMC, pode ser útil na triagem de indivíduos suscetiveis ao surgimento de preocupações excessivas com o peso corporal e a alimentação.

Palavras-chave Imagem corporal, Transtornos alimentares, Avaliação nutricional 


\section{Introdução}

O padrão estético atual de corpo difere do preconizado no início do século XX. Há uma supervalorização de um corpo magro, definido e musculoso como sinal de saúde, beleza e poder e não como imagem de desnutrição, pobreza e até mesmo doença infecciosa como no passado ${ }^{1,2}$. Esta realidade cria uma situação de frustração, baixa autoestima e discriminação entre aqueles que fogem desta regra, podendo ser esta uma condição relevante para o surgimento de transtornos alimentares $(\mathrm{TA})^{3}$.

Tem-se discutido como a mídia, a família e a moda são capazes de influenciar a percepção corporal bem como o comportamento alimentar de jovens. Becker et al. ${ }^{4}$ mostraram que o impacto da exposição televisa sobre adolescentes da ilha de Fiji apresentou associação positiva com o aumento de indicadores de transtornos alimentares e com a maior preocupação quanto à perda de peso.

Os distúrbios alimentares mais conhecidos são a anorexia nervosa e a bulimia nervosa. Ambos são caracterizados pela preocupação excessiva com o peso, principalmente por jovens do sexo feminino. Considerando a complexidade multifatorial relacionada ao aparecimento destes distúrbios, frequentemente há uma ideia negativa da imagem corporal, temor à obesidade e facilidade de adesão a diferentes métodos inadequados para o controle de peso ${ }^{5}$.

Os transtornos de comportamento alimentar têm aumentado desde as últimas décadas do século passado ${ }^{6}$, atingindo cerca de $20 \%$ de mulheres jovens adultas ${ }^{5}$. Esta prevalência aumenta para $35 \%$ entre estudantes de Nutrição ${ }^{7}$. O conhecimento da suscetibilidade ao desenvolvimento de tais doenças em estudantes da área de saúde, em especial a Nutrição, é de extrema importância. Esta profissão remete a uma preocupação constante com a aparência física e a boa forma. Este contexto, aliado ao conhecimento sobre os alimentos adquiridos ao longo do curso de Nutrição, faz com que o grupo mereça atenção por apresentar maior risco de desenvolver transtornos alimentares.

Assim, o objetivo deste trabalho foi avaliar a relação entre a alteração do comportamento alimentar, associado a insatisfação com a imagem corporal, e o estado nutricional de universitárias do Curso de Nutrição.

\section{Métodos}

Trata-se de um estudo epidemiológico transversal com universitárias do curso de Nutrição da Universidade Federal de Ouro Preto (UFOP), MG, Brasil. O protocolo do estudo foi aprovado pelo Comitê de Ética em Pesquisa da UFOP.

Todas as universitárias acima de 18 anos, regularmente matriculadas no curso foram convidadas a participar do estudo. Foram excluídos os homens e as estudantes gestantes e nutrizes. As universitárias que concordaram em participar do estudo assinaram o Termo de Consentimento Livre Esclarecido.

As estudantes preencheram um formulário com informações pessoais e responderam os questionários de Teste de Atitudes Alimentares (Eating Attitudes Test/ EAT-26) ${ }^{8}$, para avaliação de comportamento de risco para transtornos alimentares, e o Questionário de Imagem Corporal (Body Shape Questionaire-BSQ)', para avaliação com a imagem corporal.

Para o teste EAT-26, as participantes que obtiveram pontuação maior que 20 pontos foram consideradas de alto risco para do desenvolvimento de transtornos alimentares; as que apresentaram pontuação de 10 a 20 foram consideradas de baixo risco e as com escore de 0 a 9 pontos, foram consideradas isentas de risco ${ }^{8}$. Quanto ao BSQ, pontuação menor ou igual a 80 pontos foi considerada como um padrão de normalidade e tido como ausência de insatisfação com a imagem corporal. Resultados entre 81 e 110 pontos foi classificado como insatisfação leve; entre 111 e 140 como insatisfação moderada; e acima de 140 pontos foi classificada como presença de insatisfação grave com a imagem corporal ${ }^{9,10}$.

Os questionários não foram identificados, sendo codificados pela equipe de pesquisa. Sua aplicação foi feita em sala de aula, na ausência do docente, contando com a presença de dois aplicadores treinados.

A avaliação antropométrica foi realizada, em data posteriormente agendada, por um único avaliador devidamente treinado pelo coordenador da pesquisa. O peso e o percentual de gordura corporal foram determinados em balança digital de bioimpedância elétrica bipolar (TANITA Ironman Segmental Body Composition Monitor ${ }^{\circledR}$ - modelo BC554) com as voluntárias utilizando roupas leves e sem sapatos. As voluntárias foram instruídas a ingerir pelo menos dois litros de líquido além de evitar a realização de exercícios, consumo de bebidas alcoólicas e café pelo menos duas horas antes da realização das 
medidas. Para aferição da estatura foi utilizado o estadiômetro portátil AlturaExata ${ }^{\circledR}$, com escala de 35 a $213 \mathrm{~cm}$ e precisão de $0,1 \mathrm{~cm}^{11}$. O percentual de gordura corporal (\%GC) foi classificado de acordo com Lohman et al. ${ }^{12}$, sendo considerado o resultado igual ou maior que $30 \%$ como excesso de gordura corporal.

O índice de massa corporal (IMC) foi calculado pela equação de Quetelet $(\mathrm{IMC}=\mathrm{Peso} / \mathrm{Al}-$ tura $^{2}$ ) e interpretado conforme a Organização Mundial da Saúde (OMS) ${ }^{13}$.

A dobra cutânea tricipital (DCT) foi realizada no hemicorpo direito do indivíduo e sendo a dobra aferida pelo adipômetro (LANGE ${ }^{\varpi}$ ) a um centímetro abaixo do ponto pinçado pelos dedos. O resultado foi expresso em média de três medidas da mesma dobra ${ }^{13}$.

Os perímetros corporais foram realizados com o avaliador em pé e estando a fita métrica flexível em ângulo reto em relação ao eixo do cor$\mathrm{po}^{11}$. O perímetro do braço (PB) foi aferido no ponto médio entre o acrômio e o olecrano. O resultado foi interpretado conforme valores de referência ${ }^{14}$. O perímetro da cintura (PC) foi aferido com a participante em pé com a fita circundando o ponto médio entre a última costela e a crista ilíaca e leitura feita no momento da expiração ${ }^{11}$. Considerou-se como ponto de corte para PC aumentado resultado maior ou igual que $80 \mathrm{~cm}^{14}$.

\section{Análise Estatística}

Utilizou-se para a análise dos dados o programa SPSS 12.0. Para a análise estatística, considerou-se como EAT negativo (EAT-26 -) as alunas com baixo e nenhum risco para TA e positivo (EAT-26+), as alunas classificadas com alto risco para TA. Para o BSQ, considerou-se negativo (BSQ -) as universitárias que não tiveram ou apresentavam leve insatisfação com a sua imagem corporal e positivo (BSQ + ), as estudantes que apresentaram insatisfação moderada e grave.

Após avaliação da normalidade das variáveis contínuas (parâmetros antropométricos) pelo teste de Kolmogorov-Smirnov, utilizou-se o teste não paramétrico de U-Mann Whitney (IMC, DCT, \% GC, CB, PC) para comparação entre as universitárias quanto à presença de BSQ e EAT26 positivos (+) e negativos (-). A associação entre a pontuação dos questionários EAT-26 e BSQ com as medidas antropométricas foram avaliadas pelo coeficiente de Correlação de Spermann. O cálculo da razão de chances (odds ratio) estimou o risco de insatisfação com a imagem cor- poral e alteração do comportamento alimentar com a presença de excesso de peso pela classificação do IMC, aumento do \% GC e do PC. O nível de significância foi fixado em $5 \%$.

\section{Resultados}

Das 253 alunas matriculadas no curso, 175 concordaram em participar da pesquisa. A média de idade encontrada foi de 21,54 $\pm 1,91$ anos e a maioria (80,2\%) estava cursando o período ideal.

A maior parte das estudantes tinha alguma religião $(90,9 \%)$, as faixas de renda média familiar predominantes foram de 4 a 6 salários mínimos $(40,2 \%)$ e maior que 6 salários mínimos $(32,8 \%)$. Grande parte das estudantes recebia ajuda financeira dos pais $(88,3 \%)$ e $55,1 \%$ tinham algum tipo de bolsa da Universidade (iniciação científica, extensão, Programa de Educação Tutorial e monitoria).

Segundo o EAT-26; 21,7\% ( $\mathrm{n}=38)$ das estudantes apresentaram alto risco de desenvolverem transtornos alimentares, 41,7\% $(\mathrm{n}=73)$ apresentou baixo risco e 36,6\% (n=64) não apresentaram risco para TA. Quanto à pontuação do questionário de imagem corporal (BSQ), verificou-se que $63,4 \%$ das estudantes $(\mathrm{n}=111)$ não apresentaram insatisfação com a imagem corporal, $22,9 \%(n=40)$ apresentaram insatisfação leve, $8,0 \%(n=14)$ insatisfação moderada e 5,7\% $(\mathrm{n}=10)$ insatisfação grave. Associando os resultados dos dois questionários, 57,9\% $(\mathrm{n}=103)$ apresentaram EAT-26 e BSQ negativos e 16,9\% $(\mathrm{n}=30)$ EAT-26 e BSQ positivos. (Figura 1)

Das 175 alunas participantes da pesquisa, 117 realizaram a avaliação nutricional antropométrica. Segundo a classificação do IMC, 9,4\% ( $\mathrm{n}=$ 11) estavam abaixo do peso, $82,1 \%(n=96)$ estavam eutróficas, $6,8 \%(\mathrm{n}=8)$ com sobrepeso e $1,7 \%(n=2)$, com obesidade. A maioria das estudantes que apresentaram EAT-26 e BSQ positivos estava eutrófica (Tabela 1). Os valores de odds ratio indicam que as estudantes com obesidade e sobrepeso apresentaram cerca de 5 a 7 vezes mais chances de insatisfação com a imagem corporal e alto risco para transtorno alimentar do que as eutróficas. O PC elevado também aumentou as chances de insatisfação com a imagem corporal e alto risco para transtornos alimentares em 3,0 vezes. O maior \% GC elevou as chances de insatisfação com a imagem corporal e risco de transtornos alimentares em 6,45 vezes. (Tabela 1 ) 


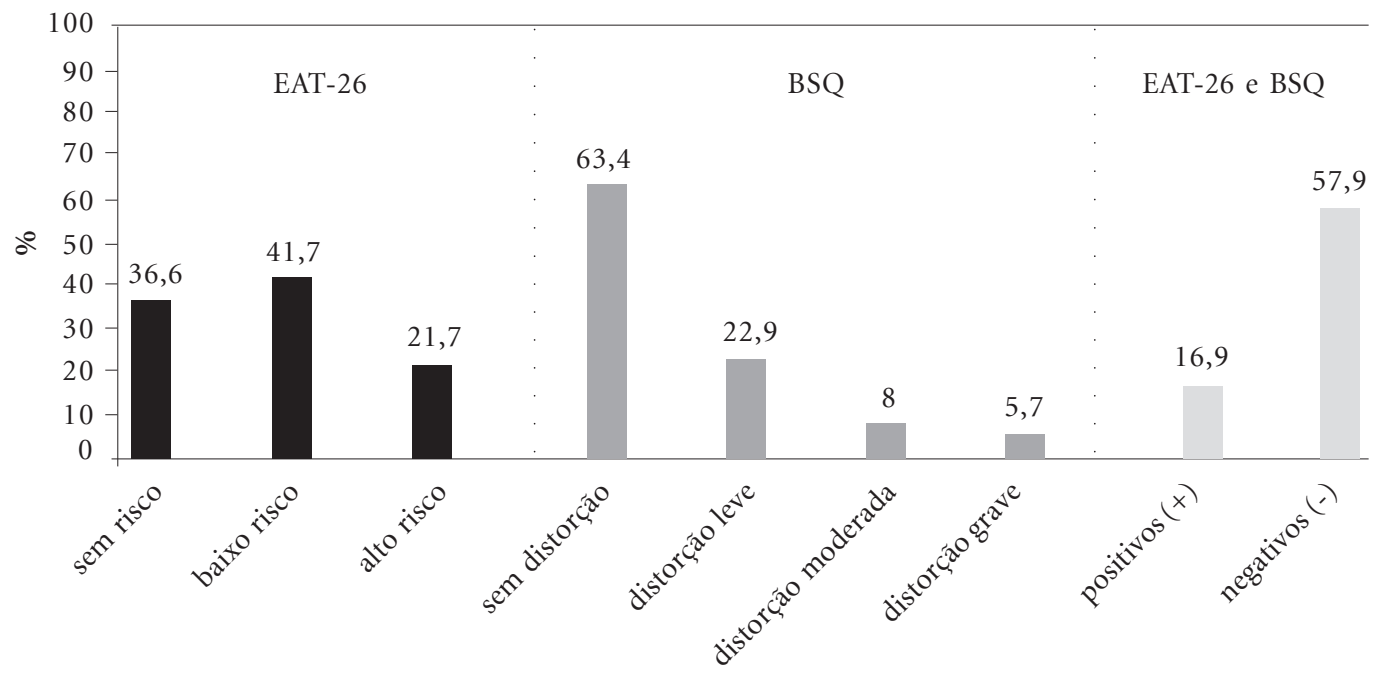

Figura 1. Percentual de universitárias que apresentaram ou não risco para transtorno alimentar (EAT-26) e alteração na imagem corporal (BSQ). EAT-26: Teste de Atitudes Alimentares (Eating Attitudes Test). BSQ: Questionário de Imagem Corporal (Body Shape Questionaire). BSQ e EAT-26 -: presença de baixo e nenhum risco para TA associado à ausência ou distorção leve da imagem corporal. BSQ e EAT +: presença de alto risco para TA associado à distorção moderada a grave da imagem corporal.

Tabela 1. Características antropométricas e percepção da imagem corporal associado ao risco de desenvolvimento de transtornos alimentares.

\begin{tabular}{|c|c|c|c|c|c|c|}
\hline & \multicolumn{2}{|c|}{ BSQ e EAT-26 - } & \multicolumn{2}{|c|}{ BSQ e EAT-26 + } & \multirow[b]{2}{*}{ Odds Ratio } & \multirow[b]{2}{*}{ Intervalo de Confiança $(p)$} \\
\hline & $\mathbf{N}$ & $\%$ & $\mathbf{N}$ & $\%$ & & \\
\hline \multicolumn{7}{|c|}{ Classificação IMC } \\
\hline Eutrofia & 67 & $(85,9)$ & 12 & $(66,7)$ & 1,00 & \\
\hline Magreza & 7 & $(9,0)$ & 1 & $(5,6)$ & 0,76 & $0,12-5,32(0,08)^{*}$ \\
\hline Sobrepeso & 3 & $(3,8)$ & 4 & $(22,2)$ & 7,11 & $1,14-47,57(0,008)^{\star}$ \\
\hline Obesidade & 1 & $(1,3)$ & 1 & $(5,5)$ & 5,33 & $0-213,30(0,20)^{\star}$ \\
\hline Total & 78 & $(100,0)$ & 18 & $(100,0)$ & & \\
\hline \multicolumn{7}{|l|}{ PC } \\
\hline Adequada & 75 & $(96,2)$ & 13 & $(72,2)$ & 1,00 & \\
\hline Aumentada & 3 & $(3,8)$ & 5 & $(27,8)$ & 9,62 & $1,70-59,51(0,0009)^{\star}$ \\
\hline Total & 78 & $(100,0)$ & 18 & $(100,0)$ & & \\
\hline \multicolumn{7}{|l|}{$\%$ GC } \\
\hline Adequado & 71 & $(91,0)$ & 11 & $(61,1)$ & 1,00 & \\
\hline Aumentado & 7 & $(9,0)$ & 7 & $(38,9)$ & 6,45 & $1,63-26,22(0,038)^{* *}$ \\
\hline Total & 78 & $(100)$ & 18 & $(100)$ & & \\
\hline
\end{tabular}

EAT-26: Teste de Atitudes Alimentares (Eating Attitudes Test-26). BSQ: Questionário de Imagem Corporal (Body Shape Questionaire). BSQ e EAT-26 -: presença de baixo e nenhum risco para TA associado a ausência ou distorção leve da imagem corporal. BSQ e EAT-26 +: presença de alto risco para TA associado a distorção moderada a grave da imagem corporal. IMC: Classificação do estado nutricional pelo índice de massa corporal; PC: Classificação do perímetro de cintura (aumentada: $\geq 80 \mathrm{~cm}$ e adequada: $<80 \mathrm{~cm})^{14}$; $\%$ GC: percentual de gordura corporal (adequado: $<30 \%$; aumentado: $\geq 30 \%$ ) ${ }^{12}$; ${ }^{*}$ Diferença estatisticamente significativa $(\mathrm{p}<0,05) ;{ }^{*}$ Valor de $p$ obtido pelo teste exato de Fisher. ${ }^{*}$ Valor de $p$ obtido pelo teste do Qui-quadrado de Pearson. 
Quando comparados os valores médios dos parâmetros antropométricos e de composição corporal, as alunas com insatisfação de moderada a grave da imagem corporal (BSQ +) apresentaram médias de IMC, \% GC, PC, PB e DCT maiores do que as alunas que não apresentaram distorção da imagem corporal (BSQ -), apesar de estarem dentro dos valores de referência para mulheres. $\mathrm{O}$ mesmo pode ser observado entre as estudantes que apresentaram alto risco para transtorno alimentar (EAT-26+). Este grupo apresentou valores médios superiores para os mesmos parâmetros $(\mathrm{p}<0,05)$. As estudantes com BSQ e EAT-26 positivos também apresentaram maiores médias dos parâmetros antropométricos relatados $(\mathrm{p}<0,05)$ (Tabela 2$)$.

Houve associação positiva significativa entre os parâmetros antropométricos e de composição corporal e a pontuação dos questionários EAT-26 e BSQ (Tabela 3).

\section{Discussão}

São poucos os estudos que relacionaram a avaliação nutricional antropométrica, com parâmetros diferentes do IMC, e a insatisfação com imagem corporal e o risco de desenvolver transtornos alimentares. O presente estudo indica que futuras nutricionistas com sobrepeso e obesidade, percentual de gordura corporal aumentado e perímetro da cintura acima dos valores de referência apresentam 5 a 9 vezes mais chances de insatisfação com a imagem corporal e alto risco de transtornos alimentares.

No presente estudo, 21,7\% das estudantes de Nutrição apresentaram risco para transtorno alimentar (EAT-26 > 20). Comparando nosso resultado com estudos disponíveis na literatura nacional, Fiattes e Salles ${ }^{15}$ observaram que 25,4\% das estudantes de Nutrição apresentaram comportamento de risco para TA. Recentemente, Alvarenga et al. ${ }^{16}$ identificaram que $26,1 \%$ das universitárias brasileiras da área da saúde vêm apresentando risco de desenvolver transtornos alimentares com preocupações anormais com a alimentação e o peso corporal ${ }^{17}$. Outros estudos indicam que as futuras nutricionistas apresentaram maior prevalência de TA ou insatisfação com a imagem corporal do que estudantes de outros cursos de graduação não relacionados à área da saúde ${ }^{15,16}$. Prevalências de alto risco de transtornos alimentares maiores do que $20 \%$ são preocupantes e apontam que bailarinos, modelos e profissionais da área da saúde, principalmente

Tabela 3. Correlação dos parâmetros antropométricos e de composição corporal em relação à pontuação dos questionários BSQ e EAT-26

\begin{tabular}{lrr}
\hline $\begin{array}{c}\text { Parâmetros } \\
\text { antropométricos }\end{array}$ & $\boldsymbol{B S Q \boldsymbol { r } ( \boldsymbol { p } )}$ & EAT-26r $(\boldsymbol{p})$ \\
\hline IMC & $0,50(0,00001)$ & $0,24(0,008)$ \\
$\%$ GC & $0,43(0,00001)$ & $0,21(0,021)$ \\
PC & $0,48(0,000001)$ & $0,38(0,009)$ \\
PB & $0,44(0,00001)$ & $0,30(0,001)$ \\
DCT & $0,29(0,001)$ & $0,18(0,048)$ \\
\hline
\end{tabular}

Índice de Massa Corporal (IMC), Percentual de gordura corporal (\%GC), Perímetro da Cintura (PC), Perímetro do Braço (PB), Dobra Cutânea Tricipital (DCT)

EAT-26: Teste de Atitudes Alimentares (Eating Attitudes Test). BSQ: Questionário de Imagem Corporal (Body Shape Questionaire)

Tabela 2. Valores médios das variáveis antropométricas de acordo com o BSQ e EAT-26 positivos e negativos das estudantes do curso de Nutrição.

\begin{tabular}{|c|c|c|c|c|c|c|}
\hline \multirow{2}{*}{$\begin{array}{c}\text { Parâmetros } \\
\text { medidos }\end{array}$} & \multicolumn{2}{|c|}{$\mathrm{BSQ}^{*}$} & \multicolumn{2}{|c|}{ EAT-26 ${ }^{* *}$} & \multicolumn{2}{|c|}{ BSQ e EAT-26 ${ }^{* * *}$} \\
\hline & $+(\mathbf{n}=36)$ & $-(n=81)$ & $+(n=21)$ & $-(n=96)$ & $+(n=18)$ & $-(n=75)^{a}$ \\
\hline $\mathrm{IMC}$ & 22,9 & 0,6 & 3,41 & 21 & $23,1 \pm 3,28$ & 20,6 \\
\hline$\%$ GC & $26,6 \pm 6,07$ & 22,0 & $26,3 \pm 7,54$ & $22,8 \pm$ & $27,2 \pm 7,44$ & 22,1 \\
\hline PB & $27,8 \pm 2,95$ & $26,4 \pm 7,93$ & $27,9 \pm 3,13$ & $26,6 \pm 7,37$ & $28,3 \pm 3,06$ & $26,5 \pm 8,07$ \\
\hline PC & $72,6 \pm 6,83$ & $67,2 \pm 7,46$ & $72,6 \pm 8,57$ & $68 \pm 7,24$ & $74,0 \pm 8,22$ & $67,3 \pm 7,51$ \\
\hline DCT & $24,1 \pm 5,92$ & $21,0 \pm 5,27$ & $23,7 \pm 5,98$ & $21,5 \pm 5,51$ & $24,5 \pm 5,96$ & $21,1 \pm 5,32$ \\
\hline
\end{tabular}

Índice de Massa Corporal (IMC), Percentual de gordura corporal (\%GC), Perímetro da Cintura (PC), Perímetro do Braço (PB), Dobra Cutânea Tricipital (DCT). EAT-26: Teste de Atitudes Alimentares (Eating Attitudes Test). BSQ: Questionário de Imagem

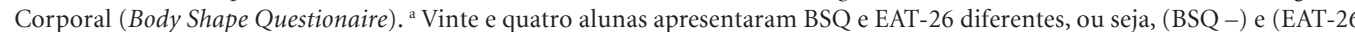
+) ou $(\mathrm{BSQ}+)$ e (EAT-26 -). ${ }^{*} \mathrm{p}<0,05$ para os valores médios dos parâmetros antropométricos quando comparados os grupos positivos e negativos para BSQ; ${ }^{* *} \mathrm{p}<0,05$ para os valores médios dos parâmetros antropométricos quando comparados os grupos positivos e negativos para EAT-26, exceto para os valores de DCT ( $>0,05) ;{ }^{* *} \mathrm{p}<0,05$ para os valores médios dos parâmetros antropométricos quando comparados os grupos positivos e negativos para BSQ e EAT-26. 
as nutricionistas, apresentam maior risco de desenvolvimento de transtorno alimentar ${ }^{16-18}$.

A análise do teste BSQ revelou que 13,7\% das universitárias estavam insatisfeitas com a sua imagem corporal. Esta prevalência é semelhante à insatisfação encontrada entre estudantes de Nutrição do Rio de Janeiro $(18,6 \%)^{1}$, Porto Alegre $(13,5 \%)^{18}$ e São Paulo $(18,5 \%)^{19}$.

Alguns autores utilizam dados referidos quanto ao peso corporal e a estatura em seus estudos ${ }^{18}$ devido à alta concordância entre a informação dada e a medição dos mesmos ${ }^{20-22}$. Entretanto, apesar de pequena, há diferença significativa entre as medidas aferidas e autorreferidas, sendo indicada a aferição quando possível ${ }^{23}$. No presente estudo, não foram questionados o peso corporal e a estatura e sim aferidos, uma vez que foram utilizados outros parâmetros antropométricos além do IMC. A realização da avaliação antropométrica permitiu ampliar a análise da possível influência do estado nutricional no comportamento alimentar.

Houve maior prevalência de eutrofia entre as universitárias que apresentaram alto risco para transtornos alimentares e insatisfação com a imagem corporal. Estudo populacional realizado em Porto Alegre com mulheres de 12 a 19 anos mostrou que a percepção do peso corporal (sentir-se gorda) representou um papel determinante no comportamento alimentar anormal, mesmo entre as participantes cujo IMC estava adequado ${ }^{6}$.

A prevalência de eutrofia entre as estudantes de Nutrição que apresentaram BSQ ou EAT-26 positivos foi de $12,5 \%(n=12 / 93)$. Prevalências maiores de $16,8 \%(n=29 / 172)^{1}$ e até de $39 \%(n$ $=60 / 154)^{7}$ foram descritas na literatura. Porém estes autores observaram a prevalência de eutrofia em relação ao resultado positivo do EAT-26 $6^{1,7}$ ou do $\mathrm{BSQ}^{1}$, isoladamente. O presente estudo apresenta um diferencial quanto aos estudos citados, pois descreve o perfil nutricional em relação à presença de positividade nos dois questionários simultaneamente (BSQ e EAT-26), o que pode ter interferido na menor prevalência.

Universitárias eutróficas e insatisfeitas com a sua imagem corporal indicam uma preocupação exagerada com a estética corporal ${ }^{24}$. É importante a abordagem dos cursos de Nutrição sobre o tema no sentido de alertar sobre a forte pressão sociocultural que impõe um ideal de corpo bonito e saudável que se sobrepõe aos princípios da saúde e da atuação do profissional nutricionista.

Este trabalho apresenta limitações de um estudo transversal, não permitindo a identificação de relações de causa e efeito. Também se limita pela desistência de 58 alunas em continuar a participação na pesquisa realizando a avaliação antropométrica. $\mathrm{O}$ desinteresse pela pesquisa foi o motivo de desistência de 21 alunas (46,7\%), 14 $(31,1 \%)$ tinham horários incompatíveis com os da avaliação, a vergonha do peso foi o motivo de desistência de oito $(17,8 \%)$ e duas $(4,4 \%)$ saíram do curso. As demais não responderam o motivo da desistência da realização da avaliação antropométrica. Possivelmente, as que não se consideravam dentro dos padrões estabelecidos pela mídia recusando-se a continuar na pesquisa.

Entretanto, com este estudo também foi possível observar que os valores médios dos parâmetros antropométricos de \% GC, PC, PB e DCT foram maiores entre as alunas com BSQ +, EAT$26+$ e BSQ e EAT-26 + em comparação com aquelas que apresentaram BSQ e/ou EAT-26 negativos. A associação positiva dos referidos parâmetros com a pontuação dos questionários BSQ e EAT-26 indica que há um aumento da pontuação destes questionários com o aumento das adiposidades abdominal (PC), corporal (\% GC) e periférica (DCT e PB). O excesso de peso e de gordura corporal aumentou as chances de insatisfação com a imagem corporal e o risco para o desenvolvimento de transtornos alimentares.

Não foram encontrados na literatura estudos que relacionam, além do IMC, outras medidas antropométricas (\% GC, PC, DCT, PB) com a insatisfação da imagem corporal e risco para transtorno alimentar entre universitários, o que inviabiliza possíveis comparações. Porém, nossos resultados com universitárias são semelhantes aos encontrados em adolescentes. Martins et al. ${ }^{25} \mathrm{ob}-$ servaram que a insatisfação com a imagem corporal apresentou associação com o estado nutricional, avaliado pelo IMC, e com a adiposidade corporal, avaliado pelo \% GC. Os autores observaram um aumento na insatisfação à medida que o IMC e o \% GC também aumentavam ${ }^{25}$.

Os resultados encontrados no presente estudo são bastante preocupantes. Mesmo apresentando medidas ou índices de avaliação do estado nutricional dentro da normalidade, é expressivo o total de universitárias do curso de Nutrição que estão insatisfeitas com o peso e as dimensões corporais e que, por isso, apresentaram risco para desenvolvimento de transtornos alimentares. Além de concordar com dados da literatura, este estudo também mostrou que as futuras nutricionistas com excesso de peso, aumento do percentual de gordura corporal e do perímetro da cintura apresentaram maior risco de estarem 
insatisfeitas com a sua imagem corporal e desenvolverem transtornos alimentares.

Além do IMC, a avaliação de outros parâmetros antropométricos pode ser bastante eficiente na triagem de indivíduos suscetíveis ao surgimento de tais, uma vez que a percepção distorcida de partes específicas do corpo também pode desencadear atitudes alimentares anormais de risco para instalação de TA.

\section{Colaboradores}

JD Silva, ABJ Silva, AVK Oliveira e ASA Nemer participaram igualmente de todas as etapas de elaboração do artigo.

\section{Agradecimentos}

À Roberta RR Álvares pelo auxílio na coleta de dados, à PROPP/UFOP pelo apoio financeiro (programas PIP e PIVIC) e à Késia Diego Quintaes, ao Marcelo Eustáquio Silva, à Cláudia Aparecida Marliére de Lima e a todos os professores da Escola de Nutrição/UFOP que colaboraram para a realização deste estudo.

\section{Referências}

1. Bosi MLM, Luis RR, Morgado CMC, Costa MLS, Carvalho RJ. Auto-percepção da imagem corporal entre estudantes de nutrição: um estudo no município do Rio de Janeiro. J Bras Psiquiatr 2006; 55(2):108-113.

2. Liao Y, Knoesen NP, Castle DJ, Tang J, Deng Y, Bookun R, Chen X, Hao W, Meng G, Liu T. Symptoms of disordered eating, body shape, and mood concerns in male and female Chinese medical students. Compr Psychiatry 2010; 51(1):516-523.

3. Kakeshita IS, Almeida SS. Relação entre índice de massa corporal e a percepção da auto-imagem em universitários. Rev Saude Publica 2006; 40(2):497-504.

4. Becker AE, Burwell RA, Herzog DB, Hamburg P, Gilman SE. Eating behaviors and attitudes following prolonged exposure to television among ethnic Fiji adolescents girls. Br J Psychiatry 2002; 180: 509-514.

5. Nunes AM, Olinto MTA, Barros FC, Camey S. Influência da percepção do peso e do índice de massa corporal nos comportamentos alimentares anormais. Rev Bras Psiquiatr 2001; 23(1):21-27.

6. Coqueiro RS, Petroski EL, Pelegrini A, Barbosa AR. Insatisfação com a imagem corporal: avaliação comparativa da associação com estado nutricional em universitários. Rev. Psiquiatr RS 2008; 30(1):31-38.

7. Penz LR, Dal Bosco SM, Vieira JM. Risco para desenvolvimento de transtornos alimentares em estudantes de Nutrição. Scientia Medica 2008; 18(3): 124-128.

8. Bighetti F, Santos CB, Santos JE, Ribeiro RPP. Tradução e validação do Eating Attitudes Test em adolescentes do sexo feminino de Ribeirão Preto-SP. J Bras Psiquiatr 2004; 53(6):339-346

9. Cooper PJ, Taylor MJ, Cooper Z, Fairbum CG. The development and validation of the body sahoe questionnaire. Int J Eat Disord 1987; 6(4):485-594.

10. Cordás TA, Neves JEP. Escalas de avaliação de transtornos alimentares. Rev Psiq Clin 1999; 26(N. Esp. 1):41-48.

11. Guedes DP. Composição corporal: princípios, técnicas e aplicações. Santa Catarina: CEITEC; 1998.

12. Lohman TG, Roche AF, Martorell R, editors. Anthropometric standardization reference manual. Champaign: Human Kinetics Books; 1988. 
13. Organização Mundial da Saúde (OMS). Obesity Presenting and managing the global epidemic. Report of a WHO consultation on obesiy. Genebra: OMS: 1998

14. Alberti KGMM, Zimmet P, Shaw J. Metabolic Syndrome - A new world-wide definition. A consensus statement from the International Diabetes Federation. Diabet Med 2006; 23(5):469-480.

15. Fiattes GMR, Salles RK. Fatores de risco para o desenvolvimento de distúrbios alimentares: um estudo em universitárias. Rev. Nutr. 2001; 14(Supl.):3-6.

16. Alvarenga MS, Scagliusi FB, Philippi ST. Comportamento de risco para transtorno alimentar em universitárias brasileiras. Rev Psiq Clin. 2011; 38(1):3-7.

17. Laus MF, Moreira RCM, Costas TMB. Diferenças na percepção da imagem corporal, no comportamento alimentar e no estado nutricional de universitárias das áreas de saúde e humanas. Rev Psiquiatr RS. 2009; 31(3):192-196.

18. Garcia CA, Castro TG, Soares RM. Comportamento alimentar e imagem corporal entre estudantes de Nutrição de uma universidade pública de Porto Alegre-RS. Rev HCPA 2010; 30(3):219-224.

19. Stipp LM, Oliveira MR. Imagem corporal e atitudes alimentares: diferenças entre estudantes de nutrição e de psicologia. Saude Rev. 2003; 5(9):47-51.

20. Fonseca MJM, Faerstein E, Chor D, Lopes CS. Validade de peso e estatura informados e índice de massa cor-poral: estudo pró-saúde. Rev Saude Publica 2004; 38(3):392-398.

21. Peixoto MRG, Benicio MHD, Jardim PCBV. Validade do peso e da estatura auto-referidos: o estudo de Goiânia. Rev Saude Publica 2006; 40(6):1065-1072.

22. Schmidt MI, Duncan BB, Tavares M, Polanczyk CA, Pellanda L, Zimmer PM. Validity of self-reported weight - A study of urban Brazilian adults. Rev Saude Publica 1993; 27(4):271-276.

23. Oliveira AGP, Oliveira TP, Lima JRP, Ferreira MEC. Diferença entre peso e estatura auto-referidos e aferidos para o cálculo do índice de massa corporal e sua relação com a imagem corporal de mulheres de academia de ginástica. HU Revista 2008; 34(3): 179-183.

24. Quadros TMB, Gordia AP, Martins CR, Silva DAS, Ferrari EP, Petroski ER. Imagem corporal em universitários: associação com o estado nutricional e sexo. Motriz Rev Ed Fis 2010; 16(1):78-85.

25. Martins CR, Pelegrini A, Matheus SC, Petroski EL. Insatisfação com a imagem corporal e relação com estado nutricional, adiposidade corporal e sintomas de anorexia e bulimia em adolescentes. Rev Psiquiatr RS 2010; 32(1):19-23.

Artigo apresentado em 03/08/2011

Aprovado em 19/12/2011

Versão final apresentada em 13/01/2011 